\title{
The High and Rising Costs of Obesity to the US Health Care System
}

\author{
Adam Biener, $P h D^{7}$, John Cawley, $P h D^{2}$, and Chad Meyerhoefer, $P h D^{3}$

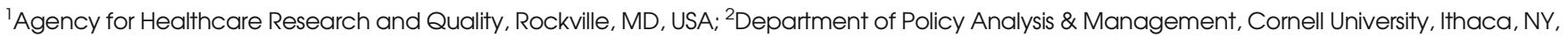 \\ USA; ${ }^{3}$ Lehigh University, Bethlehem, PA, USA.
}

J Gen Intern Med 32(Suppl 1):S6-S8

DOI: $10.1007 / \mathrm{s} 11606-016-3968-8$

(c) Society of General Internal Medicine 2017

$\mathrm{T}$ he papers in this special issue examine behavioral, pharmacotherapy, and surgical interventions for weight loss, based on a 2016 conference on state-of-the-art weight management hosted by the Veterans Health Administration. The purpose of this editorial is to provide new information on the medical care costs of obesity that help motivate those interventions.

We have a long-standing research agenda to measure the causal effect of obesity on medical care costs. The challenge is that obesity is not randomly distributed in society; as a result, the health care costs of the obese and nonobese may differ for reasons other than weight, such as differences in access to health care, income, or other dimensions of health. Our method for estimating the causal effect of obesity on medical care costs is to estimate instrumental variable (IV) models in which we exploit the heritability of weight as a natural experiment. ${ }^{1,2}$ The logic is that this variation in weight is not chosen by the individual or caused by earned income, injuries, or other events, and by estimating how medical care costs vary with such exogenous variation in weight, we can estimate the causal effect of obesity on medical care costs.

Our past research, ${ }^{1,2}$ which is based on data from the Medical Expenditure Panel Survey (MEPS), indicates that, between 2005 and 2010, the amount by which obesity raised medical costs per obese adult rose from $\$ 3070$ to $\$ 3508$ (both measured in year 2010 dollars), an increase of 14.3 percent. During that same period, the aggregate costs of obesity in the adult, noninstitutionalized population of the US rose from $\$ 212.4$ billion to $\$ 315.8$ billion (both in 2010 dollars), an increase of 48.7 percent; this large increase is due to three factors: (1) an increase in costs per obese individual; (2) an increase in the population, so even if the prevalence of obesity remained constant there would be more obese individuals; (3) an increase in the prevalence of obesity.

In this editorial we re-estimate our IV models of the impact of obesity on medical care costs using MEPS data for 20062013, that is, after adding 3 more years of data (2011-2013) and removing older years of data (2000-2005) from our

Published online March 7, 2017 sample. ${ }^{3}$ Table 1 below indicates that we estimate that obesity raises the medical care costs of obese adults by an average of $\$ 3429$ (in 2013 dollars); this is slightly less than the point estimate for 2010 ( $\$ 3748$ in 2013 dollars) $)^{2}$, but the difference is not statistically significant.

Those statistics describe the difference in average expenditures for obese and non-obese individuals, adjusted for sociodemographic factors. However, there may be interesting nonlinearities in how medical care costs vary along the range of body mass index (BMI). To explore this, we estimate an IV model in which medical care costs are regressed on BMI and BMI squared. Fig. 1 plots predicted medical care costs over BMI for men and women in the MEPS 2006-2013 as the solid line; the dashed lines indicate the 90 percent confidence intervals. The figure shows that expenditures have a J-shape over BMI, i.e., expenditures fall with BMI through the categories of underweight $(\mathrm{BMI} \leq 18.5)$ and healthy weight $(18.5<\mathrm{BMI}<$ 25 ), are relatively constant with BMI in the overweight range $(25 \leq \mathrm{BMI}<30)$, and then rise slowly with BMI through the range of obese class I $(30 \leq \mathrm{BMI}<35)$ and then rise rapidly in the range of obese class II $(35 \leq \mathrm{BMI}<40)$ and especially obese class III $(40 \leq \mathrm{BMI})$.

Under the assumption that the effect of obesity in our subpopulation generalizes to the full non-institutionalized population of adults aged 18 and older, we estimate that total medical costs for the full non-institutionalized population of adults aged 18 and older in the US in 2013 were $\$ 342.2$ billion. Comparing these new results to those from our earlier papers $^{1,2}$, the share of total health care spending of noninstitutionalized adults that is devoted to treating obesityrelated illness has risen from 20.6 percent in 2005 to 27.5 percent in 2010 to 28.2 percent in 2013.

These estimates of the medical care costs of obesity are useful for several reasons. First, they are necessary to calculate the cost-effectiveness of various anti-obesity treatments, interventions, and policies. Our causal estimates of the effect of obesity on medical care costs tend to be larger than estimates of the correlation of obesity with medical care costs that are estimated using models that do not correct for the endogeneity of weight. ${ }^{1}$ Cost-effectiveness studies that use estimates of the correlation of obesity with medical care costs, rather than the causal effect, may underestimate the benefits of preventing and reducing obesity, which could result in suboptimal investment in obesity prevention and treatment.

Second, this information is useful for targeting weight loss programs. The dotted line in the figure shows the distribution 
Table 1 Increases in annual individual medical expenditures due to obesity and a one unit increase in BMI for different groups of adults with biological children

\begin{tabular}{|c|c|c|c|c|}
\hline \multirow[t]{2}{*}{ Sample } & \multicolumn{2}{|c|}{ Total expenditures } & \multicolumn{2}{|c|}{ Third party expenditures } \\
\hline & BMI \$ (SE) & Obesity \$ (SE) & BMI \$ (SE) & Obesity \$ (SE) \\
\hline Total $(\mathrm{N}=31,591)$ & $197(43)$ & $3429(799)$ & $180(41)$ & $3210(793)$ \\
\hline Males $(\mathrm{N}=12,349)$ & $244(94)$ & 3565 (1437) & $225(87)$ & 3395 (1381) \\
\hline Females $(\mathrm{N}=19,242)$ & $179(59)$ & $3359(804)$ & $164(38)$ & $3116(783)$ \\
\hline White $(\mathrm{N}=12,895)$ & $144(59)$ & 2557 (1173) & $134(54)$ & 2454 (1084) \\
\hline Non-White $(\mathrm{N}=18,696)$ & $261(71)$ & $4086(1050)$ & $240(70)$ & 3799 (1034) \\
\hline Private insurance $(\mathrm{N}=19,384)$ & $216(53)$ & 3846 (1077) & $199(51)$ & $3581(1041)$ \\
\hline Medicaid ( $\mathrm{N}=4464)$ & $182(102)$ & 2954 (1711) & $170(96)$ & 2809 (1633) \\
\hline Uninsured $(\mathrm{N}=7152)$ & $91(51)$ & $1689(922)$ & N/A & N/A \\
\hline
\end{tabular}

Notes: Standard errors are adjusted for the complex design of the MEPS, and all dollar values are reported in 2013 USD. Bolded estimates are statistically significant at $\alpha=0.05$. Data: MEPS, 2006-2013

of BMI in the MEPS sample. This, in conjunction with the solid line that shows expected expenditures by BMI unit, indicates that it is a very small percentage of the sample at high levels of BMI that incurs a disproportionate share of obesity-related medical costs. Thus, the relevant comparison is not the obese to the non-obese, but the morbidly obese (i.e., those of class II and III obesity) to everyone else. The figure also suggests that there are no savings in medical care costs that can be achieved by targeting weight loss interventions to the overweight and few savings from targeting them to people with class I obesity. Addressing the high health care costs of obesity requires focusing on the relatively small fraction of adults who are morbidly obese (i.e., have class II or III obesity). This suggests a potentially important role for bariatric surgery; see the articles by Funk et al. and Peterson in this issue. $^{4,5}$

Third, this information is useful for public policy. The second set of columns in the table lists the medical care costs of obesity that are paid by third-party payers, which include health insurance companies and public health insurance. These represent "external" costs of obesity - costs paid by society as a whole, as opposed to obese individuals. According to the Table, $\$ 3210$

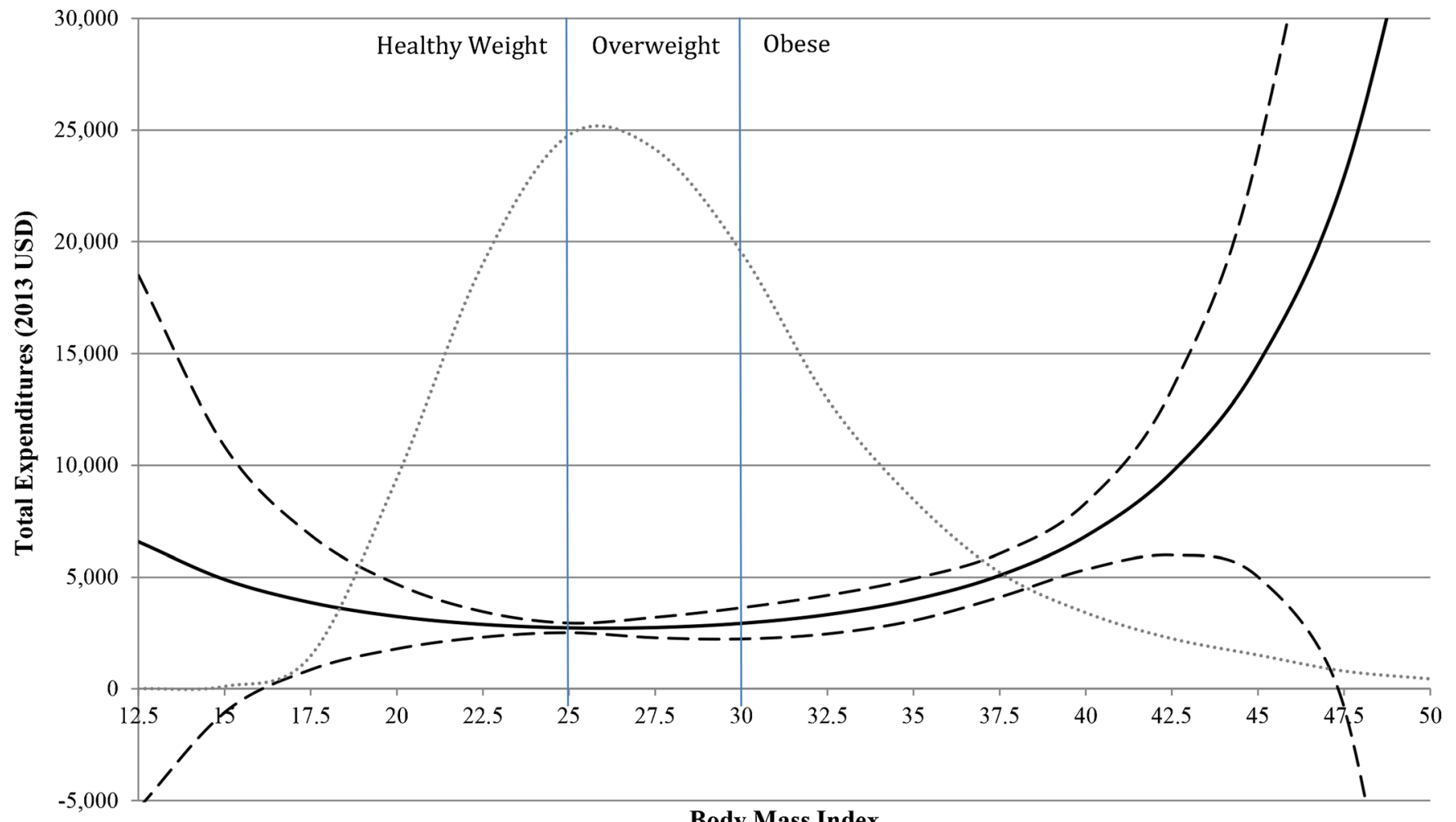

Body Mass Index

Notes: Predicted medical expenditures (in 2013 USD) are denoted by the solid line. Dashed lines represent the $90 \%$ confidence interval, which has been adjusted for the complex design of the MEPS. The distribution of individuals in the population is indicated by the dotted line. BMI is calculated using self-reports or proxy reports of weight and height. Data: MEPS, 2006-2013.

Fig. 1 Predicted relationship between BMI and annual medical expenditures for all adults with biological children 
out of the $\$ 3429$, or 93.6 percent, of the increase in medical expenditures due to obesity is incurred by third-party payers and thus represents negative externalities of obesity. These external costs represent an important economic rationale for government intervention to prevent and reduce obesity. ${ }^{6}$

The results we present are subject to the following caveats and limitations. As with any application of IV, one must be cautious that the instrument is valid; that is, the instrument should not affect the outcome (here, medical care costs) except through the endogenous regressor (here, BMI). In our IV model, the instrument for adult BMI is the BMI of their biological child. A potential threat to the validity of this instrument is pleiotropy: the genes that affect weight may also affect things that affect residual medical care costs; however, a study of the genes linked to obesity found that they tend to only be related to obesityrelated outcomes. ${ }^{7}$ Still, one can never prove the null hypothesis of no correlation between the instrument and unobserved factors. A second limitation of the analysis is that the MEPS contains only self-reported and not measured weight and height, and reporting error can lead to bias in coefficient estimates. ${ }^{8}$

Despite these limitations, these new, updated estimates of the medical care costs of adult obesity have important implications, for both the Veteran's Administration (VA) specifically and the health care sector more broadly. The VA manages the health care of individuals at all points in the BMI distribution; for example, for certain overweight and obese veterans it offers the MOVE! weight management program ${ }^{9}$, weight loss medications, and bariatric surgery. Based on Fig. 1, those transiting into class II obesity (BMI $\geq 35)$ are expected to incur rapidly escalating health care costs with BMI. The VA could identify individuals approaching this threshold and prioritize them for interventions that prevent further weight gain. The expected benefits of implementing such interventions can be estimated using Fig. 1. The VA can compare these expected benefits to the cost of weight loss drugs to evaluate their criteria for use, and the VA could also use these estimates to determine BMI cutoffs for bariatric surgery based on its costeffectiveness at different points in the BMI distribution.

These estimates of the medical care costs of adult obesity also have implications beyond the VA. For example, the high external costs represent an economic rationale for government intervention to prevent and reduce obesity, and Fig. 1, displaying estimated medical care costs by BMI, can be used by health insurers, employers, and government agencies to determine the cost-effectiveness of, and business case for, interventions that prevent and reduce obesity.

Corresponding Author: John Cawley, PhD; Department of Policy Analysis \& Management Cornell University 2312 MVR Hall, Ithaca NY 14853, USA (e-mail: JHC38@cornell.edu).

\section{Compliance with Ethical Standards:}

Funders: Cawley thanks the Robert Wood Johnson Foundation for their support through an Investigator Award in Health Policy Research.

Prior Presentations: None.

Disclaimer: The views expressed in this article are those of the authors and do not necessarily represent those of the Agency for Healthcare Research and Quality or the US Department of Health and Human Services.

Conflict of Interest: The authors declare no conflicts of interest.

\section{REFERENCES}

1. Cawley J, Meyerhoefer C. The medical care costs of obesity: an instrumental variables approach. J Health Econ. 2012;31(1):219-30.

2. Cawley J, Meyerhoefer C, Biener A, Hammer M, Wintfeld N. Savings in medical expenditures associated with reductions in body mass index among adults with obesity, by diabetes status. Pharmacoeconomics. 2015;33:707-22.

3. Specifically, we estimated two-part IV models of health care costs in which the BMI of a biological child serves as an instrument for the BMI of the adult MEPS respondent. Given the nature of the instrument, the MEPS sample is limited to adults who have a biological child living in their household. The age restriction for adults is 20-64 years and that for biological children is 11-20 years. For more details on the models and data, please see Cawley and Meyerhoefer (2012) and Cawley, Meyerhoefer, and Biener (2015).

4. Funk LM, Gunnar W, Eisenberg D, et al. A health services research agenda for bariatric surgery within the Veterans Health Administration. This issue.

5. Peterson $\mathbf{K}$. Rapid evidence review of bariatric surgery in super obesity (BMI $>50 \mathrm{~kg} / \mathrm{m} 2)$. J Gen Intern Med. 2016. doi:10.1007/s11606-0163950-5.

6. Cawley J. An economy of scales: a selective review of obesity's economic causes, consequences, and solutions. J Health Econ. 2015;43:244-68.

7. Speliotes EK, Willer CJ, Berndt SI, et al. Association analyses of 249,796 individuals reveal 18 new loci associated with body mass index. Nat Genet. 2010;42:937-48.

8. Cawley J, Maclean JC, Hammer M, Wintfeld N. Reporting error in weight and its implications for estimates of the economic consequences of obesity. Econ Human Biol. 2015; 19:27-44.

9. U.S. Department of Veterans Affairs. MOVE! Weight Management Program. http://www.move.va.gov/QandA.asp Accessed 12 Dec 2016. 